\title{
On-Chip Fabrication of Cell-Attached Microstructures using Photo-Cross-Linkable Biodegradable Hydrogel
}

\author{
Masaru Takeuchi ${ }^{1, *(D)}$, Taro Kozuka ${ }^{2}$, Eunhye Kim ${ }^{2}{ }^{(D}$, Akihiko Ichikawa ${ }^{2}$, \\ Yasuhisa Hasegawa ${ }^{1}$, Qiang Huang ${ }^{2}$ and Toshio Fukuda ${ }^{2,3}$ \\ 1 Department of Micro-Nano Mechanical Science and Engineering, Nagoya University, \\ Nagoya 4648603, Japan; yasuhisa.hasegawa@mae.nagoya-u.ac.jp \\ 2 Department of Mechatronics Engineering, Meijo University, Nagoya 4688502, Japan; \\ 140447024@ccmailg.meijo-u.ac.jp (T.K.); kim@meijo-u.ac.jp (E.K.); ichikawa@meijo-u.ac.jp (A.I.); \\ qhuang@bit.edu.cn (Q.H.); tofukuda@meijo-u.ac.jp (T.F.) \\ 3 Intelligent Robotics Institute, School of Mechatronical Engineering, Beijing Institute of Technology, \\ Beijing 100081, China \\ * Correspondence: takeuchi@mein.nagoya-u.ac.jp; Tel.: +81-52-789-2717
}

Received: 18 November 2019; Accepted: 10 March 2020; Published: 15 March 2020

\begin{abstract}
We developed a procedure for fabricating movable biological cell structures using biodegradable materials on a microfluidic chip. A photo-cross-linkable biodegradable hydrogel gelatin methacrylate (GelMA) was used to fabricate arbitrary microstructure shapes under a microscope using patterned ultraviolet light. The GelMA microstructures were movable inside the microfluidic channel after applying a hydrophobic coating material. The fabricated microstructures were self-assembled inside the microfluidic chip using our method of fluid forcing. The synthesis procedure of GelMA was optimized by changing the dialysis temperature, which kept the GelMA at a suitable $\mathrm{pH}$ for cell culture. RLC-18 rat liver cells (Riken BioResource Research Center, Tsukuba, Japan) were cultured inside the GelMA and on the GelMA microstructures to check cell growth. The cells were then stretched for 1 day in the cell culture and grew well on the GelMA microstructures. However, they did not grow well inside the GelMA microstructures. The GelMA microstructures were partially dissolved after 4 days of cell culture because of their biodegradability after the cells were placed on the microstructures. The results indicated that the proposed procedure used to fabricate cell structures using GelMA can be used as a building block to assemble three-dimensional tissue-like cell structures in vitro inside microfluidic devices.
\end{abstract}

Keywords: microfabrication; cell culture; microfluidics; biodegradable material; photo cross-linkable material

\section{Introduction}

Recently, cell-assembly technologies for tissue engineering and organs-on-a-chip have gained increasing attention [1-3] with the development of pluripotent stem cells [4,5]. Although artificial organs and related transplant technologies have improved, the side effects arising from defective artificial organs require further examination. Tissue engineering technology has matured rapidly recently [6-8], enabling organs to be artificially grown from stem cells.

Various assemblies of three-dimensional (3D) cell structures have been proposed in the literature. For example, two-dimensional (2D) cell sheets can be used to create thicker cell structures by picking them up and placing them layer by layer $[9,10]$. However, thicker structures are difficult to achieve this way, because the thickness can only be several tens of microns. To achieve 3D cell structures of larger size, robotics technologies have been applied. For example, a bio-printing method based on inkjet 
technologies was recently developed [11-15] to increase the fabrication speed and the strength of cell structures. Hydrogels (e.g., alginates) have been employed to eject and encapsulate cells inside 3D structures. However, fabrication speeds are limited, especially when the fabricated structure becomes larger, because the 3D printer must fabricate 3D structures from one-dimensional dots (i.e., droplets).

Fluidic assembly is a suitable approach for the high-throughput assembly of microstructures, including biological objects [16]. For example, complex 3D structures have been assembled by cell-encapsulated blocks [17]. Tubular structures made of biological cells have been assembled using a self-assembly process [18]. Different 3D microstructures have been also assembled inside microfluidic devices by self-assembly process. Hence, micro-assembly inside a microfluidic device shows great potential for high-throughput self-assembly of 3D cellular structures $[19,20]$.

A photo-cross-linkable resin was employed to encapsulate cells for 3D structures using self-assembly process inside a microchannel $[1,21]$. In these studies, 3D structures were fabricated from $2 \mathrm{D}$ cell structures, revealing the potential to achieve higher assembly efficiencies compared with bio-printing. However, non-biodegradable materials were generally employed for a photo-cross-linkable resin. For this study, we developed a procedure for fabricating movable biological cell structures using a biodegradable material in a microfluidic chip, in which cells are cultured on microstructures to achieve 2D cell structures. The fabricated microstructures are movable and can be used as building blocks for 3D assembly of in vitro cell structures.

The main contributions of this study are the achievement of cell cultures on biodegradable hydrogel microstructures and the assembly demonstration of movable hydrogel microstructures inside a microfluidic device. The fabricated microstructures with cells can be used as building blocks for 3D tissue-like cell structures. The assembly of microstructures can be conducted inside the microfluidic chip using a self-assembly process described previously [1]. A demonstration of assembling GelMA microstructures inside the two-layered microchannel was conducted. The fabrication and assembly procedures require microrobotics technologies of microfabrication and micromanipulation.

\section{Materials and Methods}

\subsection{Preparation of Biodegradable Microstructures}

The GelMA was synthesized as follows [22-25]. First, type-A porcine skin gelatin was added at $10 \% \mathrm{w} / \mathrm{v}$ to phosphate-buffered saline (PBS) and heated at $50{ }^{\circ} \mathrm{C}$ for $1 \mathrm{~h}$ with stirring. Methacrylic anhydride (Sigma-Aldrich Japan, Tokyo, Japan) was then added $(7.5 \% \mathrm{v} / \mathrm{v})$ to the gelatin solution at around $60{ }^{\circ} \mathrm{C}$ while stirring, allowing it to react for $2 \mathrm{~h}$. In synthesized methacrylated gelatin, gelatin macromers containing primary amino groups were reacted using methacrylic anhydride to add methacrylate pendant groups, as shown in Figure 1a. The samples were then dialyzed using 12-14 kDa cutoff dialysis tubing (Spectra/Por(R), Repligen, Waltham, MA, USA) in pure water for 1 week. Finally, the solution was frozen overnight $\left(-80^{\circ} \mathrm{C}\right)$, lyophilized for 1 week in a freeze-dryer (FDU-1200, Eyela, Tokyo, Japan), and stored at $-80^{\circ} \mathrm{C}$ until further use. The lyophilized GelMA was a white porous foam, as shown in Figure 1c. The lyophilized GelMA was dissolved in PBS or culture medium to fabricate GelMA microstructures.

\subsection{Cell Culture in GelMA Microstructures}

The GelMA solution was exposed to UV light to fabricate GelMA microstructures, and the light was measured. In our experiment, the UV power source (U-HGLGPS, Olympus, Tokyo, Japan) had a wavelength from $350 \mathrm{~nm}$ to $750 \mathrm{~nm}$, and the output power could be changed to six different percentages: $3 \%, 6 \%, 12 \%, 25 \%, 50 \%$, and $100 \%$. To check the fabrication conditions, the UV power at the fabrication area of microstructure was measured. The UV light from the UV power source was exposed to the fabrication area using the $60 \times$ objective lens. 


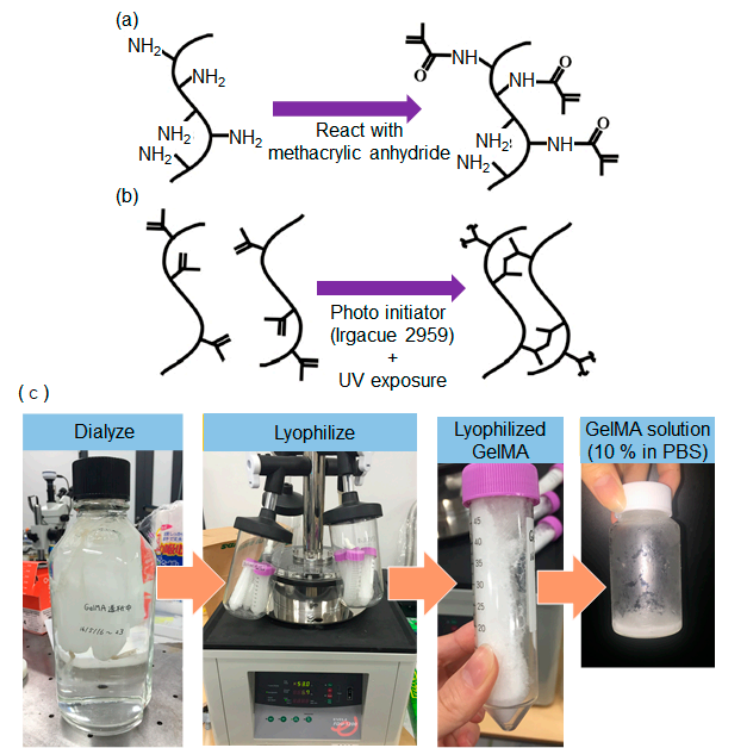

Figure 1. GelMA synthesis: (a) chemical reaction with methacrylic anhydride; (b) chemical reaction during UV exposure; (c) procedure of GelMA synthesis.

The cell culture was created inside the GelMA microstructures via embedding. Figure 2 shows the experimental procedure used to fabricate cells embedded the GelMA microstructures. In the experiments, three different types of cells were used: rat liver cells (RLC-18), mouse fibroblast cells (NIH3T3), and mouse smooth muscle cells (SMCs) (Riken BioResource Research Center, Tsukuba, Japan). To fabricate microstructures, cells were mixed into the $10 \%$ GelMA $+0.5 \%$ photo initiator (PI) solution. Irgacure 2959 was used as the photo initiator (PI). The UV light was then shone through the $60 \times$ objective lens and the mask of the toroidal shape. The GelMA solution with cells was exposed to UV light for $10 \mathrm{~s}$. The solution around the microstructures was then replaced in the culture medium (Dulbecco's modified Eagle medium (DMEM) $+10 \%$ fetal bovine serum (FBS)). The cells around the GelMA microstructures were removed from the culture dish during solution replacement. The cells inside the GelMA microstructures were then cultured inside a $37^{\circ} \mathrm{C} \mathrm{CO}_{2}$ incubator.

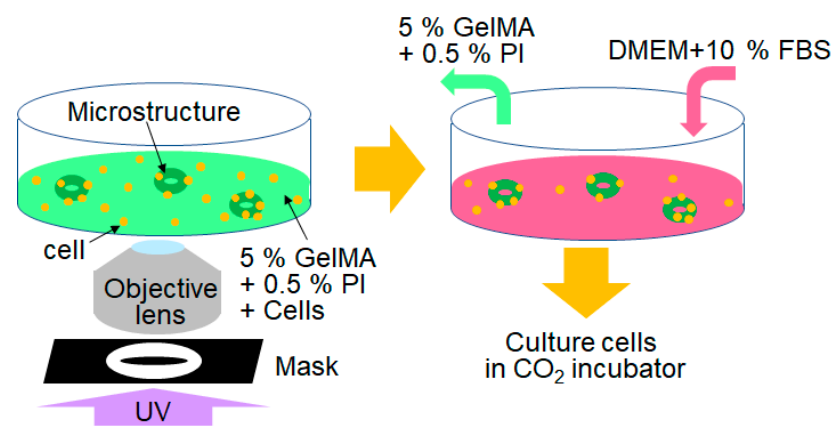

Figure 2. Fabrication of cell-embedded microstructures using GelMA.

To check cell viability of the cell culture inside the GelMA microstructures, a live/dead assay was conducted. Calcein acetoxymethyl ester (Calcein-AM) (Life Technologies, Thermo Fisher Scientific Japan, Tokyo, Japan) was mixed into the culture medium at $0.2 \mathrm{~g} / \mathrm{mL}$, and the cells were cultured for $15 \mathrm{~min}$ in the $5 \% \mathrm{CO}_{2}$ incubator. After 3 days of cell culture inside the GelMA, propidium iodide (Life Technologies, Thermo Fisher Scientific Japan, Tokyo, Japan) was used to check for dead cells. This was mixed into the culture medium at $2.5 \mathrm{~g} / \mathrm{mL}$, and cells were cultured for $5 \mathrm{~min}$. 


\subsection{Cell Culture on GelMA Surface}

In our experiment, the UV power source could output power at six different percentages: $3 \%, 6 \%$, $12 \%, 25 \%, 50 \%$, and $100 \%$. To check the fabrication conditions, the UV power at the fabrication area of the microstructure was measured. The fabrication area was exposed to the UV light through the $60 \times$ objective lens.

To check the cell growth on the GelMA microstructures, a cell culture was conducted. In the experiment, rat liver cells (RLC-18) were used. First, toroidal microstructures were fabricated as follows. The lyophilized GelMA was dissolved in the culture medium (DMEM + 10\% FBS) to make a concentration of $5 \% \mathrm{w} / \mathrm{v}$ GelMA solution. The PI powder was then dissolved in dimethyl sulfoxide (DMSO) solvent to obtain a concentration of $50 \% \mathrm{w} / \mathrm{v}$ as the stock solution. Before fabricating the GelMA structures, the experimental solution was prepared by mixing the 5\% w/v GelMA solutions and $50 \% \mathrm{w} / \mathrm{v}$ PI solution. The PI concentration became $0.5 \% \mathrm{w} / \mathrm{v}$ in the experimental solution. A mask of toroidal shape was then used to fabricate the toroidal shapes of the microstructures.

Figure 3 shows the experimental procedure of cell culture on GelMA microstructures. First, we prepared 5\% GelMA solution with $0.5 \% \mathrm{w} / \mathrm{v}$ PI solution placed inside an ultra-low attachment dish (corning). The ultra-low attachment dish was then used to prevent cell adhesion around the GelMA microstructures. The dish was then exposed to UV light for $10 \mathrm{~s}$ through the mask. After the solidification of GelMA, uncured GelMA solution was replaced with PBS, and then with a culture medium (DMEM + 10\% FBS) and rat liver cells (RLC-18) to seed the GelMA microstructures. The cells on the GelMA microstructures were then cultured inside a $37{ }^{\circ} \mathrm{C} 5 \mathrm{CO}_{2}$ incubator. In the experiments, four different UV exposure durations $(20,30,45$, and $60 \mathrm{~s}$ ) were tested for fabrication of the GelMA microstructures.

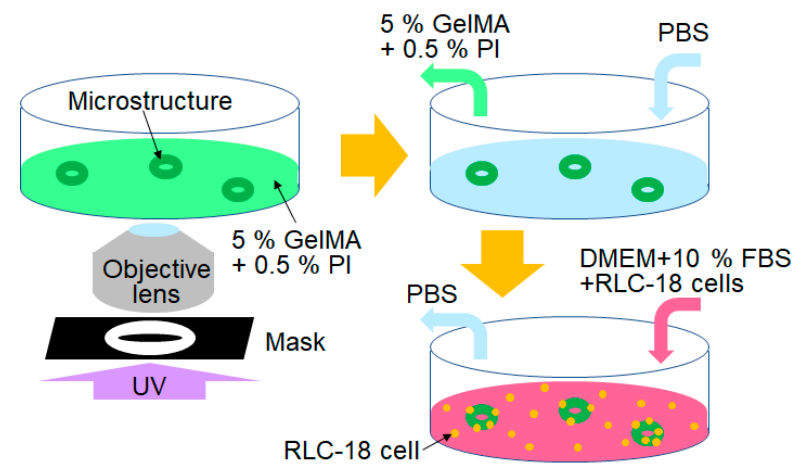

Figure 3. Experimental procedure of cell culture on GelMA microstructures.

\section{Results}

\subsection{Preparation of Biodegradable Microstructures}

A photo-cross-linkable and biodegradable gelatin methacrylate (GelMA) hydrogel was synthesized. During GelMA synthesis, the dialysis temperature is an important parameter. Figure 4 shows the GelMA synthesized at different dialysis temperatures: $40^{\circ} \mathrm{C}$ and room temperature $\left(\sim 20^{\circ} \mathrm{C}\right)$. As shown in Figure $4 a, b$, there was no significant difference in their appearance after lyophilization. However, the acid levels differed. Figure $4 \mathrm{c}, \mathrm{d}$ shows the color of the culture medium with phenolsulfonphthalein when the lyophilized GelMA was dissolved into the culture medium. The phenolsulfonphthalein changed its color depending on the $\mathrm{pH}$. The color was red when the $\mathrm{pH}$ was higher than 8.0, but the color became yellow when the $\mathrm{pH}$ was lower than 6.6. Generally, cells need to be cultured in a culture medium at around $\mathrm{pH} 8$ (red color conidtion of phenolsulfonphthalein-containing culture medium). When the GelMA was dialyzed at $40^{\circ} \mathrm{C}$, the dissolved GelMA culture medium became red. However, the dissolved GelMA culture medium became yellow (Figure $4 \mathrm{~d}$ ) and its $\mathrm{pH}$ was about 4 when the GelMA was dialyzed at room temperature. Notably, excess methacrylic acid in the GelMA 
was removed during dialysis so that the dialysis could be conducted at $40^{\circ} \mathrm{C}$. The viscosity of GelMA can be decreased by heating at $40^{\circ} \mathrm{C}$ because the main material of GelMA is gelatin, and the dialysis process can be promoted by low viscosity of the GelMA. The dissolved GelMA culture medium after dialysis at $40^{\circ} \mathrm{C}$ showed a red color (Figure $4 \mathrm{c}$ ), and its $\mathrm{pH}$ was about 8 .

(a)

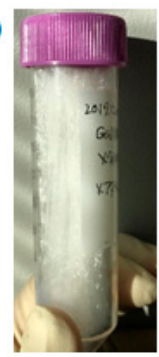

(c)

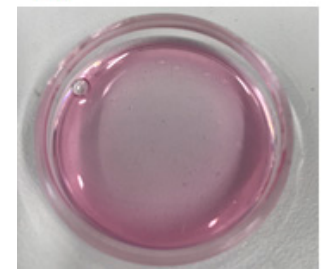

(b)

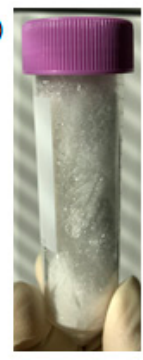

(d)

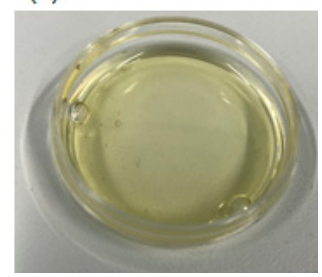

Figure 4. GelMA synthesis under different conditions: (a) lyophilized GelMA, dialyzed at $40{ }^{\circ} \mathrm{C}$; (b) lyophilized GelMA, dialyzed at room temperature; (c) dissolved GelMA in a culture medium dialyzed at $40{ }^{\circ} \mathrm{C}$; and (d) dissolved GelMA in a culture medium dialyzed at room temperature.

\subsection{Cell Culture in GelMA Microstructures}

In our method [22], cells were mixed in GelMA solution and encapsulated inside the GelMA microstructures by exposing them to patterned UV radiation. Such on-chip fabrication can be achieved by using patterned UV light through a mask. However, when the cells are encapsulated inside GelMA microstructures, only a few cells are stretched and grown [22]. In this study, we created cell cultures inside and on the GelMA microstructures to check the differences of cell growth.

To fabricate GelMA microstructures, UV power is important. Figure 5 shows the results of the UV power measurement. The UV power increased nearly proportionally with the output percentages. The relationship between UV output power, $P(\%)$, and the measured UV power, $E\left(\mathrm{~mW} / \mathrm{cm}^{2}\right)$, in our experimental setup could be expressed using linear approximation.

$$
E=0.194 P \text {. }
$$

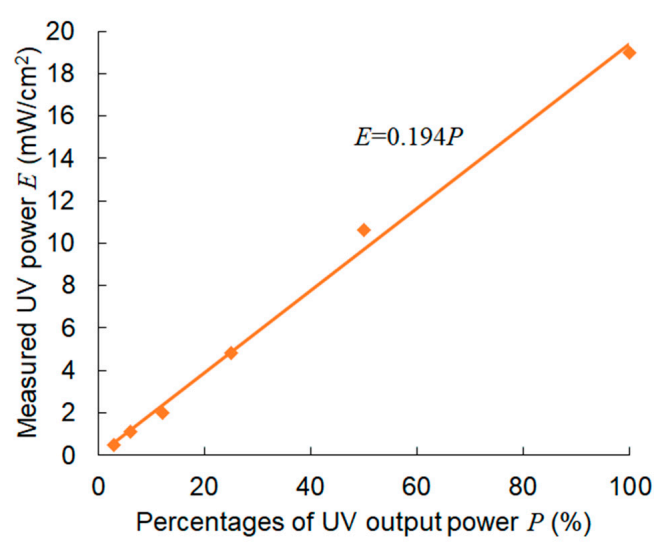

Figure 5. Measurement of UV power when a $60 \times$ objective lens was used. 
When we fabricated the GelMA microstructures, UV exposure was set between 10 and $60 \mathrm{~s}$, which corresponded to 190 and $1140 \mathrm{~mJ} / \mathrm{cm}^{2} \mathrm{UV}$ power, respectively. Thus, $10 \mathrm{~s}$ was the minimum duration to solidify the solution.

Figure 6a shows the experimental cell culture results from three cell types (i.e., rat liver cells RLS-18, mouse fibroblast NIH3T3, and mouse smooth muscle cells SMCs) after fabricating toroidal GelMA microstrucrtures using 10 s UV irradiation. In the case of RLC-18 and NIH3T3, cell shapes showed almost no change during the 3 days of cell culture, and cell growth was not observed. In the case of SMC, a few stretched cells were observed. However, most cells maintained a round shape, and the cell numbers did not increase. The results indicated that the stretched cells may not have been fully embedded inside the GelMA solution and were instead attached on the surface. However, the round cells were fully embedded in the GelMA microstructures and could not be well stretched.

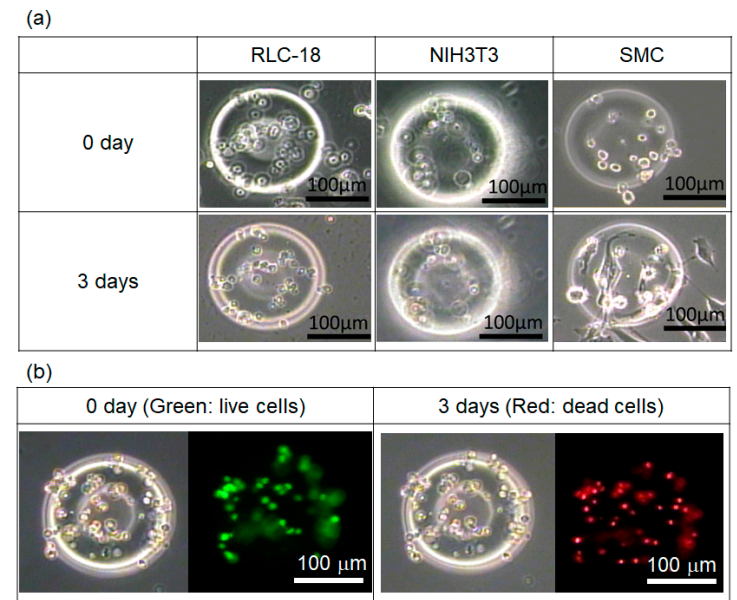

Figure 6. Cell culture in GelMA microstructures: (a) culture results using different cell types and (b) live/dead assay of RLC-18 cells in GelMA microstructure.

A live/dead assay was conducted to check cell viability in the GelMA microstructures. This was accomplished by using fluorescent dyes, calcein-AM and propidium iodide. Calcein-AM stains only live cells green fluorescent, and propidium iodide stains only dead cells red fluorescent. Just after the fabrication of cell-embedded GelMA microstructures, calcein-AM was used to check for live cells. The experimental results are shown in Figure 6b. Many cells remained alive just after the fabrication of the GelMA microstructure. However, most cells were dead after 3 days of cell culture. The results indicated that the environment inside the GelMA was not suitable for cell growth in our experimental setup.

\subsection{Cell Culture on GelMA Surface}

To enhance cell growth, the fabrication procedure of the cell microstructure was changed. In the new fabrication procedure, the GelMA microstructures were fabricated first, without cells. Cells were seeded onto the fabricated GelMA microstructures. Thus, the cells could be attached and stretched on the surface of the GelMA microstructures.

Figure 7 shows the results of culturing SMCs on the GelMA microstructures. After 1 day of cell culture, cells were attached onto the surface of GelMA microstructures, and some were stretched in all conditions. After 3 days, the cell numbers had increased, and the surfaces of GelMA microstructures were covered by cells. After 20 and 30 s UV irradiation, the GelMA microstructures were deformed because of the stretching force from the cells. However, the shape of the GelMA microstructures stayed round in cases of 45 and $60 \mathrm{~s}$ UV irradiation. The results indicated that the longer UV irradiation increased the mechanical strength of the GelMA, and that the microstructures therefore kept their shapes even when the cells covered the surface. 


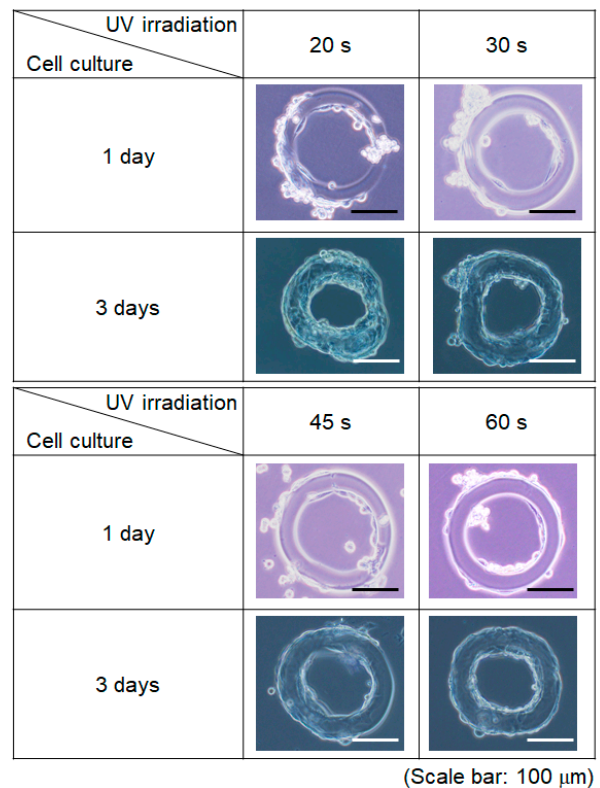

Figure 7. Cell culture on GelMA microstructure after 2 days of different UV exposures.

The degradation of the GelMA microstructures was checked using RLC-18 cells. In the experiment, cell numbers were decreased to visualize the GelMA degradation. Figure 8 shows the experimental results. In this experiment, two different sizes of microstructures were fabricated by changing the mask size, with 170 and $280 \mathrm{~m}$ outer diameters. Just after the fabrication ( 0 day), all structures were adhered to the substrate, showing a clear edge. Some microstructures were deformed and degraded after 4 days. The speed of degradation appeared to be different according to the number of cells on the microstructure.
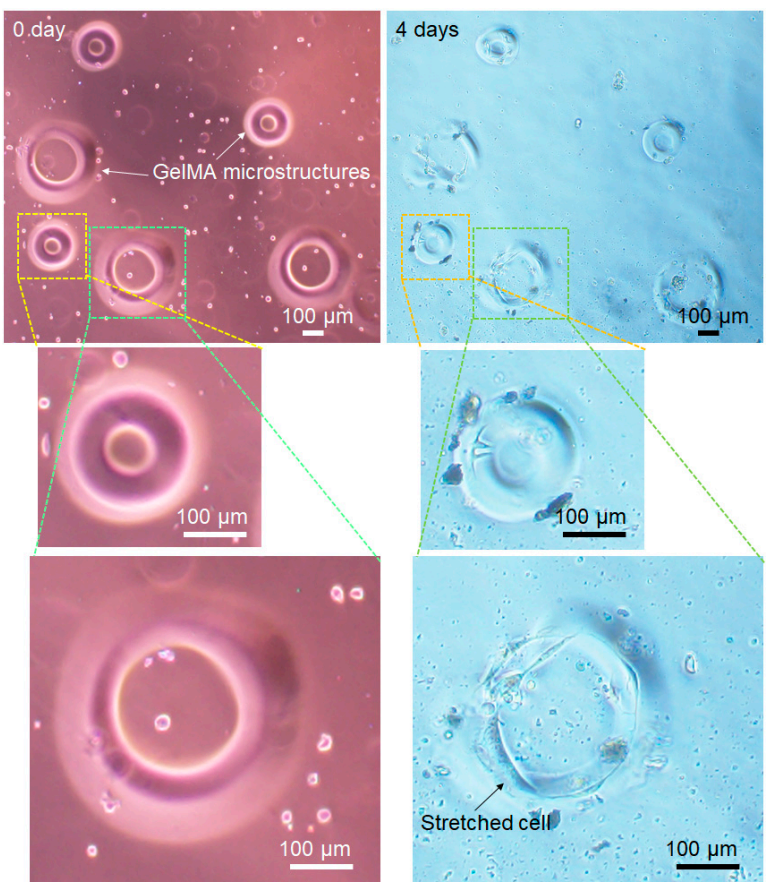

Figure 8. Degradation of GelMA during cell culture. Just after the fabrication (0 day), all structures were adhered to the substrate, showing a clear edge. Larger microstructures had larger number of cells on it and they showed deformation and degradation after 4 days, while smaller microstructures maintained a clear edge after 4 days. 
Smaller toroidal structures on the left side (magnified at the center in Figure 8) maintained a relatively clear edge compared with the larger toroidal microstructures of the smaller microstructure. The larger toroidal microstructure (magnified in the bottom of Figure 8 ) had larger number of cells on it. The results indicated that the cells on the GelMA microstructures could be cultured according to the GelMA patterns. Additionally, the GelMA could be dissolved by the cell culture because of its biodegradable character.

\subsection{Movable GelMA Microstructures in a Microfluidic Device}

In our method, 3D cell structures were fabricated using GelMA. Figure 9 shows the fabrication procedure of tubular cell structures using a photo-cross-linkable biodegradable hydrogel. In our method, $2 \mathrm{D}$ toroidal microstructures were used to assemble 3D tube shapes. The fabricated microstructures were movable when the water-repellent coating was applied to the surface of the microchannel [22]. The fabrication and assembly of microstructures could thus be conducted using the same microfluidic chip. The assembled GelMA can be degraded, resulting in only the cell structures remaining in the microfluidic chip.

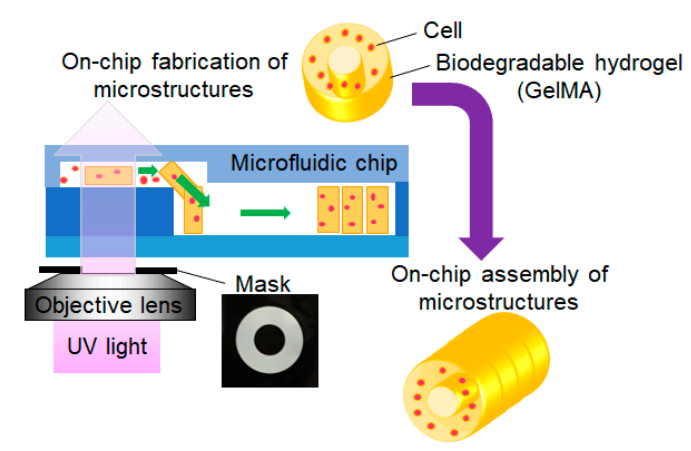

Figure 9. Fabrication procedure of tubular microstructures using on-chip fabrication and the assembly of toroidal cell-embedded microstructures.

Figure 10 shows the fabrication of movable GelMA microstructures using a microfluidic chip. Figure 10a shows the experimental setup used to check the movability of GelMA microstructures. The inlet and outlet of the microfluidic chip were connected to syringe pumps via silicon tubes. The fabrication area was exposed to UV light via a mask and an objective lens. The microfluidic chip was made of polydimethylsiloxane (PDMS) and fabricated using a general soft-lithography process. The channel was coated by a water-repellent coating material to peel off the microstructures after fabrication [22]. In the microchannel, toroidal GelMA microstructures were fabricated using patterned UV light. After fabrication, the syringe pumps were turned on, and the flow in the microchannel generated enough force to peel off the fabricated microstructures. As shown in Figure 10b,c, two different sizes of GelMA microstructures were moved through the microchannel by controlling the flow rate of the syringe pumps. Thus, these types of movable microstructures can be used as building blocks for 3D assembly. By choosing toroidal shapes, the assembled microstructures can then be formed into tube shapes, as shown in Figure 8.

Figure 11 shows the experimental result of self-assembly of GelMA microstructures inside the two-layered microfluidic device. In the experiment, a two-layered microchannel designed as shown in Figure 11a was used. The toroidal-shaped GelMA microstructures were flowed inside the upper layer first, and a syringe pump connected to the outlet of the microchannel was used to suck the solution inside the microchannel. The two GelMA microstructures were moved inside the channel, and they were rotated $90^{\circ}$ at the connection between upper and bottom layer of the channel as shown in Figure 11b. They were finally stacked inside the bottom-layered channel. Hence, the experimental results showed that the GelMA microstructures could be used to construct tube-shaped cell structures using our self-assembly method inside a microchannel. 

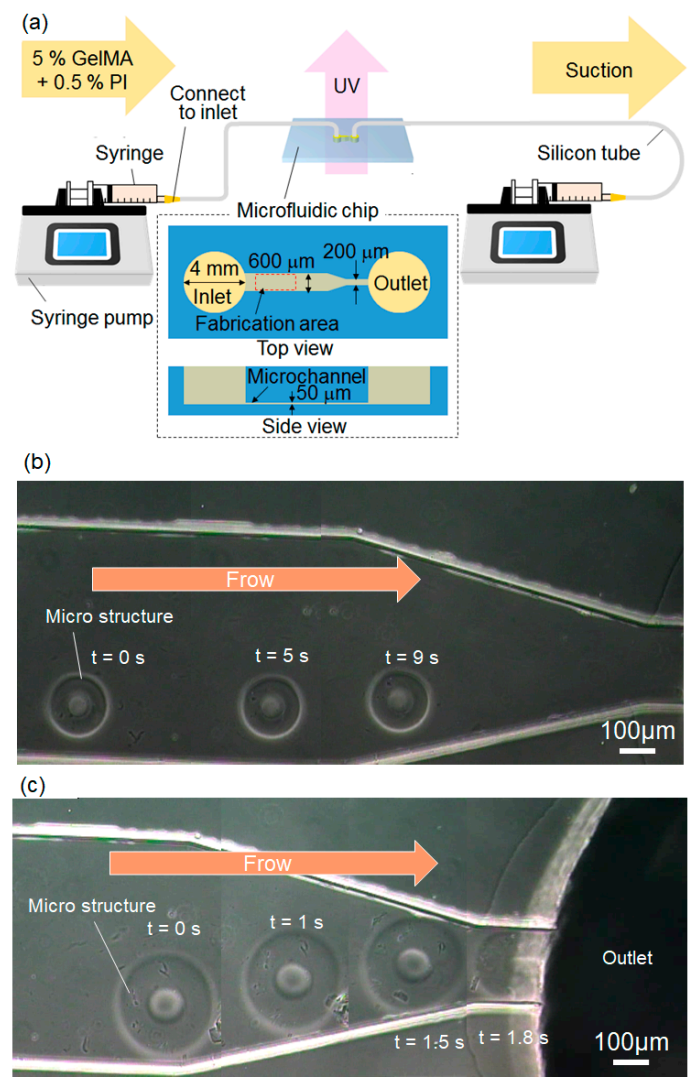

Figure 10. On-chip fabrication of movable GelMA microstructures: (a) experimental setup; (b,c) movable microstructures inside the microfluidic chip.

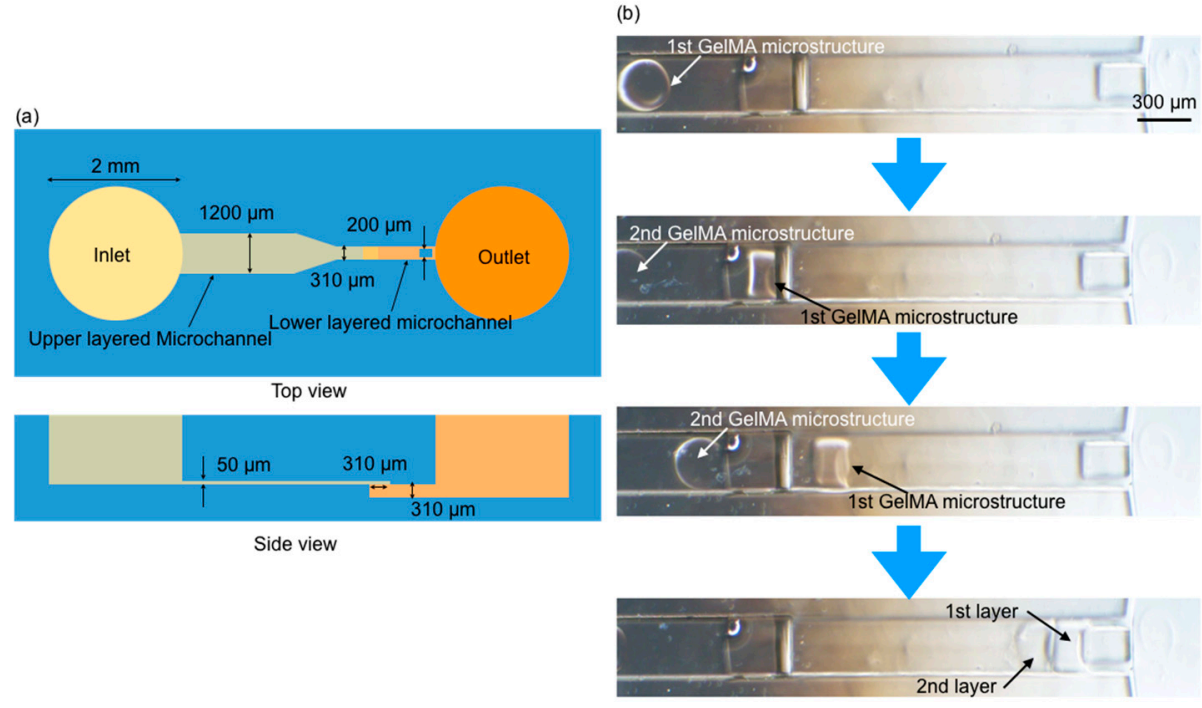

Figure 11. On-chip assembly of movable GelMA microstructures: (a) design of two-layered microfluidic device; (b) experimental results of two-layered assembly of GelMA microstructures.

\section{Conclusions}

We created a cell culture with GelMA microstructures. The biodegradable hydrogel GelMA was employed to fabricate cell microstructures. The synthesis procedure of GelMA was changed to achieve suitable conditions. The temperature of dialysis was important for removing excess methacrylic acid inside the GelMA. We experimentally confirmed that the cell-embedded conditions were not suitable for cell culture. 
The cells were then seeded on the surface of GelMA microstructures to enhance cell growth. Rat liver cells were stretched on the GelMA microstructures, and nearly the entire surface of the GelMA was covered with cells after 3 days. Partial degradation of the GelMA microstructures was observed after 4 days of cell culture. The degradation speed depended on the cell numbers attached to the surface of the GelMA. The pattern of GelMA microstructures can be transferred to the shape of cell structures using our proposed method. The fabricated toroidal microstructures were successfully manipulated in the two-layered microfluidic chip to be assembled into a tube shape. The results indicate that the GelMA microstructures can be used as a building blocks of 3D in vitro tissue-like cell structures.

Recently, 3D bioprinting technology has become one of the most promising techniques for the construction of 3D cellular structures. However, bioprinting requires a longer time to construct larger structures, since they have to be constructed from 0D (dot) to 3D (volume). Our method can be used to assemble 2D microstructures using a self-assembly process. A tube shape fabricated inside a microfluidic device using the self-assembly process can be used as a part of vascular networks. Vascular-like channel structures are necessary for constructing large 3D cell structures without necrosis. Generally, capillary networks (5-10 $\mu \mathrm{m}$ diameter) can be constructed automatically inside cell structures via the self-organization of cells when the vascular endothelial cells are properly mixed and cultured. However, artificial blood vessels larger than $6 \mathrm{~mm}$ have already been developed and clinically used. Thus, relatively larger blood vessels of several hundred micrometers have not been prepared. The method demonstrated in this paper can be used to fabricate tube shapes with the diameters required to construct relatively large 3D cell structures. Thus, the patterning of different cells in one structure is possible using our method [22].

Author Contributions: Conceptualization, M.T. and T.F.; methodology, M.T. and A.I.; validation, M.T., E.K. and T.K.; investigation, M.T., A.I.; data curation, T.K. and M.T; writing-original draft preparation, M.T.; writing-review and editing, Y.H., Q.H. and T.F.; visualization, M.T. and T.K.; supervision, T.F.; project administration, M.T. and T.F.; funding acquisition, T.F. All authors have read and agreed to the published version of the manuscript.

Funding: This work was supported in part by the Ministry of Education, Culture, Sports, Science and Technology of Japan (Grans-in-Aid for Scientific Research (A) (19H00751)).

Conflicts of Interest: The authors declare no conflict of interest. The funders had no role in the design of the study; in the collection, analyses, or interpretation of data; in the writing of the manuscript, or in the decision to publish the results.

\section{References}

1. Yue, T.; Nakajima, M.; Takeuchi, M.; Hu, C.; Huang, Q.; Fukuda, T. On-chip self-assembly of cell embedded microstructures to vascular-like microtubes. Lab Chip 2014, 14, 1151-1161. [CrossRef] [PubMed]

2. Chumtong, P.; Kojima, M.; Ohara, K.; Mae, Y.; Horade, M.; Akiyama, Y.; Yamato, M.; Arai, T. Design and fabrication of changeable cell culture mold. J. Robot Mechatron. 2013, 25, 657-664. [CrossRef]

3. Utoa, K.; Tsui,J.H.; De Forest, C.A.; Kim, D.H. Dynamically tunable cell culture platforms for tissueengineering and mechanobiology. Prog. Polym. Sci. 2017, 65, 53-82. [CrossRef] [PubMed]

4. Thomson, J.A.; Itskovitz-Eldor, J.; Shapiro, S.S.; Waknitz, M.A.; Swiergiel, J.J.; Marshall, V.S.; Jones, J.M. Embryonic stem cell lines derived from human blastocysts. Science 1998, 282, 1145-1147. [CrossRef]

5. Takahashi, K.; Yamanaka, S. Induction of pluripotent stem cells from mouse embryonic and adult fibroblast cultures by defined factors. Cell 2006, 126, 663-676. [CrossRef]

6. Vacanti, C.A. The history of tissue engineering. J. Cell. Mol. Med. 2006, 10, 569-576. [CrossRef]

7. Bedian, L.; Villalba-Rodríguez, A.M.; Hernández-Vargas, G.; Parra-Saldivar, R.; Iqbal, H.M.N. Bio-based materials with novel characteristics for tissue engineering applications-A review. Int. J. Biol. Macromol. 2017, 98, 837-846. [CrossRef]

8. Anderson, C.W.; Boardman, N.; Luo, J.; Park, J.; Qyang, Y. Stem cells in cardiovascular medicine: The road to regenerative therapies. Curr. Cardiol. Rep. 2017, 19, 34. [CrossRef] 
9. Haraguchi, Y.; Shimizu, T.; Sasagawa, T.; Sekine, H.; Sakaguchi, K.; Kikuchi, T.; Sekine, W.; Sekiya, S.; Yamato, M.; Umezu, M.; et al. Fabrication of functional three-dimensional tissues by stacking cell sheets in vitro. Nat. Protoc. 2012, 7, 850-858. [CrossRef]

10. Yamagishi, Y.; Masuda, T.; Matsusaki, M.; Akashi, M.; Yokoyama, U.; Arai, F. Microfluidic perfusion culture system for multilayer artery tissue models. Biomicrofluidics 2014, 8, 064113. [CrossRef]

11. Sasagawa, T.; Shimizu, T.; Sekiya, S.; Haraguchi, Y.; Yamato, M.; Sawa, Y.; Okano, T. Design of prevascularized three-dimensional cell-dense tissues using a cell sheet stacking manipulation technology. Biomaterials 2010, 31, 1646-1654. [CrossRef] [PubMed]

12. Nishiyama, Y.; Nakamura, M. Development of a Three-Dimensional Bioprinter: Construction of cell supporting structures using hydrogel and state-of-the-art inkjet technology. J. Biomech. Eng. 2009, 131, 035001. [CrossRef] [PubMed]

13. Cui, H.; Nowicki, M.; Fisher, J.P.; Zhang, L.G. 3D Bioprinting for organ regeneration. Adv. Healthc. Mater. 2017, 6, 1601118. [CrossRef] [PubMed]

14. Park, J.H.; Jang, J.; Lee, J.S.; Cho, D.W. Three-dimensional printing of tissue/organ analogues containing living cells. Ann. Biomed. Eng. 2017, 45, 180-194. [CrossRef] [PubMed]

15. Wüst, S.; Müller, R.; Hofmann, S. Controlled positioning of cells in biomaterials-Approaches towards 3D tissue printing. J. Funct. Biomater. 2011, 2, 119-154. [CrossRef]

16. Whitesides, G.M.; Grzybowski, B. Self-assembly at all scales. Science 2002, 295, 2418-2421. [CrossRef]

17. Kato-Negishi, M.; Morimoto, Y.; Onoe, H.; Takeuchi, S. Millimeter-Sized Neural Building Blocks for 3D Heterogeneous Neural Network Assembly. Adv. Healthc. Mater. 2013, 2, 1564-1570. [CrossRef]

18. Gauvin, R.; Ahsan, T.; Larouche, D.; Levesque, P.; Dube, J.; Auger, F.A.; Nerem, R.M.; Germain, L. A Novel Single-Step Self-Assembly Approach for the Fabrication of Tissue-Engineered Vascular Constructs. Tissue Eng. Part A 2010, 16, 1737-1747. [CrossRef]

19. Khoo, H.S.; Lin, C.; Huang, S.-H.; Tseng, F.-G. Self-Assembly in Micro- and Nanofluidic Devices: A Review of Recent Efforts. Micromachines 2011, 2, 17-48. [CrossRef]

20. Yue, T.; Liu, N.; Liu, Y.; Peng, Y.; Xie, S.; Luo, J.; Huang, Q.; Takeuchi, M.; Fukuda, T. On-Chip Construction of Multilayered Hydrogel Microtubes for Engineered Vascular-Like Microstructures. Micromachines 2019, 10, 840. [CrossRef]

21. Norotte, C.; Marga, F.S.; Niklason, L.E.; Forgacs, G. Scaffold-free vascular tissue engineering using bioprinting. Biomaterials 2009, 30, 5910-5917. [CrossRef] [PubMed]

22. Takeuchi, M.; Nakamura, Y.; Ichikawa, A.; Hasegawa, A.; Hasegawa, Y.; Fukuda, T. On-chip fabrication of movable toroidal cell structures using photo-crosslinkable biodegradable hydrogel. In Proceedings of the 2017 IEEE/RSJ International Conference on Intelligent Robots and Systems (IROS 2017), Vancouver, BC, Canada, 24-28 September 2017.

23. Ramón-Azcón, J.; Ahadian, S.; Obregón, R.; Camci-Unal, G.; Ostrovidov, S.; Hosseini, V.; Kaji, H.; Ino, K.; Shiku, H.; Khademhosseini, A. Gelatin methacrylate as a promising hydrogel for 3D microscale organization and proliferation of dielectrophoretically patterned cells. Lab Chip 2012, 12, 2959-2969. [CrossRef] [PubMed]

24. Nichol, J.W.; Koshy, S.T.; Bae, H.; Hwang, C.M.; Yamanlar, S.; Khademhosseini, A. Cell-laden microengineered gelatin methacrylate hydrogels. Biomaterials 2011, 31, 5536-5544. [CrossRef] [PubMed]

25. Chen, Y.C.; Lin, R.Z.; Qi, H.; Yang, Y.; Bae, H.; Melero-Martin, J.M.; Khademhosseini, A. Functional human vascular network generated in photocrosslinkable gelatin methacrylate hydrogels. Adv. Funct. Mater. 2012, 22, 2027-2039. [CrossRef] [PubMed]

(C) 2020 by the authors. Licensee MDPI, Basel, Switzerland. This article is an open access article distributed under the terms and conditions of the Creative Commons Attribution (CC BY) license (http://creativecommons.org/licenses/by/4.0/). 